\title{
SÍNDROME DE HAMMAN: NEUMOMEDIASTINO ESPONTÁNEO QUE DEBUTA COMO ENFISEMA CERVICOTORÁCICO MASIVO. DESCRIPCIÓN DE UN CASO
}

\section{Hamman's Syndrome: spontaneus pneumomediastinum presenting as cervicothoracic massive emphysema. A case report}

\author{
Fernando GARCÍA-CURDI ${ }^{1}$; Mariano Andrés LÓPEZ-VÁZQUEZ ${ }^{1}$; Yolanda LOIS-ORTEGA ${ }^{1}$; Rocío EZPELE- \\ TA-BADENAS ${ }^{2}$; Jessica LÓPEZ-BUIL ${ }^{1}$; Guillermo GIL-GRASA ${ }^{1}$; Pablo VELA-GAJÓN ${ }^{1}$; Héctor VALLÉS-VA- \\ RELA $^{1}$ \\ Hospital Clínico Universitario Lozano Blesa. 1Servicio de Otorrinolaringología. 2Unidad de Enfermería del Bloque \\ Quirúrgico Zaragoza. España.
}

Correspondencia:fgcurdi@gmail.com

Fecha de recepción: 19 de marzo de 2018

Fecha de aceptación: 30 de marzo de 2018

Fecha de publicación: 2 de abril de 2018

Fecha de publicación del fascículo: 1 de junio de 2019

Conflicto de intereses: Los autores declaran no tener conflictos de intereses

Imágenes: Los autores declaran haber obtenido las imágenes con el permiso de los pacientes

Política de derechos y autoarchivo: se permite el autoarchivo de la versión post-print (SHERPA/RoMEO)

Licencia CC BY-NC-ND. Licencia Creative Commons Atribución-NoComercial-SinDerivar 4.0 Internacional

Universidad de Salamanca. Su comercialización está sujeta al permiso del editor

RESUMEN: Introducción: El neumomediastino espontáneo (NME) consiste en la presencia de aire en el mediastino sin una causa conocida. El principal síntoma del NME es el dolor torácico, que se acompaña de un enfisema cervical en el 70\% de los casos. Descripción: Presentamos el caso clínico de un paciente que acude a nuestro centro con clínica de disnea de reposo de varias horas de evolución, así como un enfisema cervicotorácico masivo. Al realizar pruebas de imagen se visualiza la presencia de aire en el mediastino. A pesar de ser tratado de manera precoz, días después falleció. Conclusiones: El NME es una enfermedad de evolución generalmente benigna, de difícil diagnóstico y que, por tanto, debe incluirse en el diagnóstico diferencial de aquellos pacientes que sufren dolor torácico asociado a disnea. Por lo general, se requiere un tratamiento conservador con observación y seguimiento, aunque en ocasiones pueden surgir complicaciones graves. 


\section{SÍNDROME DE HAMMAN: NEUMOMEDIASTINO ESPONTÁNEO QUE DEBUTA COMO ENFISEMA CERVICOTORÁCICO MASIVO. DESCRIPCIÓN DE UN CASO GARCÍA-CURDI F ET AL}

PALABRAS CLAVE: Síndrome de Hamman; neumomediastino espontáneo.

SUMMARY: Introduction: Spontaneous pneumomediastinum (NME) consists of the presence of air in the mediastinum without a known cause. The main symptom of NME is chest pain, which is accompanied by cervical emphysema in $70 \%$ of cases. Case description: We present the clinical case of a patient who comes to our center with dyspnea at rest for several hours, as well as massive cervicothoracic emphysema. When carrying out image tests, the presence of air in the mediastinum is visualized. Despite being treated early, days later he dies. Conclusions: The NME is a disease of generally benign evolution, difficult to diagnose and that, therefore, should be included in the differential diagnosis of those patients who suffer chest pain associated with dyspnea. In general, conservative treatment with observation and follow-up is required, although serious complications can sometimes arise.

KEYWORDS: Hamman’s Syndrome; spontaneous pneumomediastinum.

\section{INTRODUCCIÓN}

El síndrome de Hamman o neumomediastino espontáneo (NME) fue descrito por Louis Hamman en 1939 como «enfisema mediastínico espontáneo» [1]. Corresponde a la presencia de aire libre en el mediastino sin relación con un traumatismo torácico, procedimientos traqueobronquiales o esofágicos, ventilación mecánica, cateterización cardiaca ni cirugía torácica [2]. Ante el aumento de la presión intratorácica -como ocurre al realizar una maniobra de Valsalva-, puede producirse una rotura alveolar y diseminación de aire hacia el espacio intersticial y el mediastino (Efecto Macklin) [3]. La incidencia es aproximadamente de 1/25.000, suele afectar a pacientes con edades comprendidas entre los 5 y 35 años, y predominantemente al sexo masculino respecto al femenino con una relación 4:1. La incidencia puede ser algo mayor, ya que algunos pacientes son diagnosticados erróneamente de ansiedad o dolor muscular. Las manifestaciones clínicas más frecuentes son dolor torácico, disnea y enfisema subcutáneo [2].

\section{DESCRIPCIÓN}

Presentamos el caso de un paciente varón de 81 años que acudió al servicio de urgencias de nuestro hospital por disnea de reposo de 10 horas de evolución. Como antecedentes personales cabe destacar una alveolitis alérgica extrínseca y una enfermedad pulmonar obstructiva crónica de 4 años de evolución, lo que le generaba una insuficiencia respiratoria crónica. El paciente se encontraba consciente, orientado y con una saturación de $\mathrm{O}_{2}$ al $93 \%$ a pesar de recibir oxigenoterapia a través de gafas nasales. En la exploración otorrinolaringológica se observó un enfisema subcutáneo cervicotorácico masivo, que se extendía desde región maxilar y occipital hasta región pectoral y escapular. Ante este hallazgo se realizó una rinofibrolaringoscopia que resultó anodina. Se interrogó al paciente acerca de si había recibido algún traumatismo o si realizaba esfuerzos por tos en los últimos días, hechos que negó.

Se solicitó una tomografía computarizada (TC) cervicotorácica de urgencia, en el que se visualizó un extenso enfisema subcutáneo que disecaba todos los planos musculares del cuello y tórax, asociado a un neumomediastino severo sin observarse colecciones asociadas. No se observaron neumotórax, fracturas costales ni derrame pleural. No se apreciaron soluciones de continuidad en tráquea o esófago. Mostraba extensas áreas de fibrosis pulmonar ya conocida (Figura 1). Ante el diagnóstico de neumomediastino de causa espontánea, asociado a enfisema cervical subcutáneo fue valorado por el cirujano torácico de guardia quien consideró que no requería ninguna actuación de urgencia, por lo que ingresó en el servicio de cuidados intensivos para vigilar evolución. 


\section{SÍNDROME DE HAMMAN: NEUMOMEDIASTINO ESPONTÁNEO QUE DEBUTA COMO ENFISEMA CERVICOTORÁCICO MASIVO. DESCRIPCIÓN DE UN CASO GARCÍA-CURDI F ET AL}

Horas más tarde, tras estabilización del proceso, fue trasladado a la planta de neumología, donde en los días posteriores sufrió un empeoramiento de su insuficiencia respiratoria crónica con decaimiento del estado general, que progresó hasta el exitus.

\section{DISCUSIÓN}

El neumomediastino o la presencia de aire en el mediastino fue descrito por primera vez por Laennec en 1819, en relación con traumatismos torácicos. No obstante, en la literatura médica se encuentra un caso de enfisema subcutáneo posparto descrito por Simmons en 1783, probablemente el primer caso de NME del que se tenga conocimiento. No fue hasta 1939 cuando Louis Hamman informa los primeros casos de enfisema subcutáneo asociado a neumomediastino, fecha desde la que se acuña el término síndrome de
Hamman [2]. En 1944 Macklin y Macklin describieron el proceso fisiopatológico en el que la rotura alveolar es la responsable del neumomediastino (efecto Macklin) [4]. Con la rotura de los alveolos se produce la disección de las vainas broncovasculares y se produce una diseminación del enfisema intersticial hacia el mediastino. El efecto Macklin se origina generalmente a partir de un traumatismo torácico, aunque en ocasiones puede ocurrir de manera espontánea, dando lugar a un NME [5]. Finalmente, el aire puede extenderse hacia los tejidos subcutáneos de la pared torácica y del cuello, siguiendo los planos fasciales, originando un enfisema subcutáneo $[6,7]$. El principal síntoma del NME es el dolor torácico. Es acompañado en el $70 \%$ de los casos de un enfisema cervical, y otros síntomas como disnea, fiebre, disfagia o rinolalia [3]. El NME es un proceso de evolución generalmente benigna, y que suele no diagnosticarse

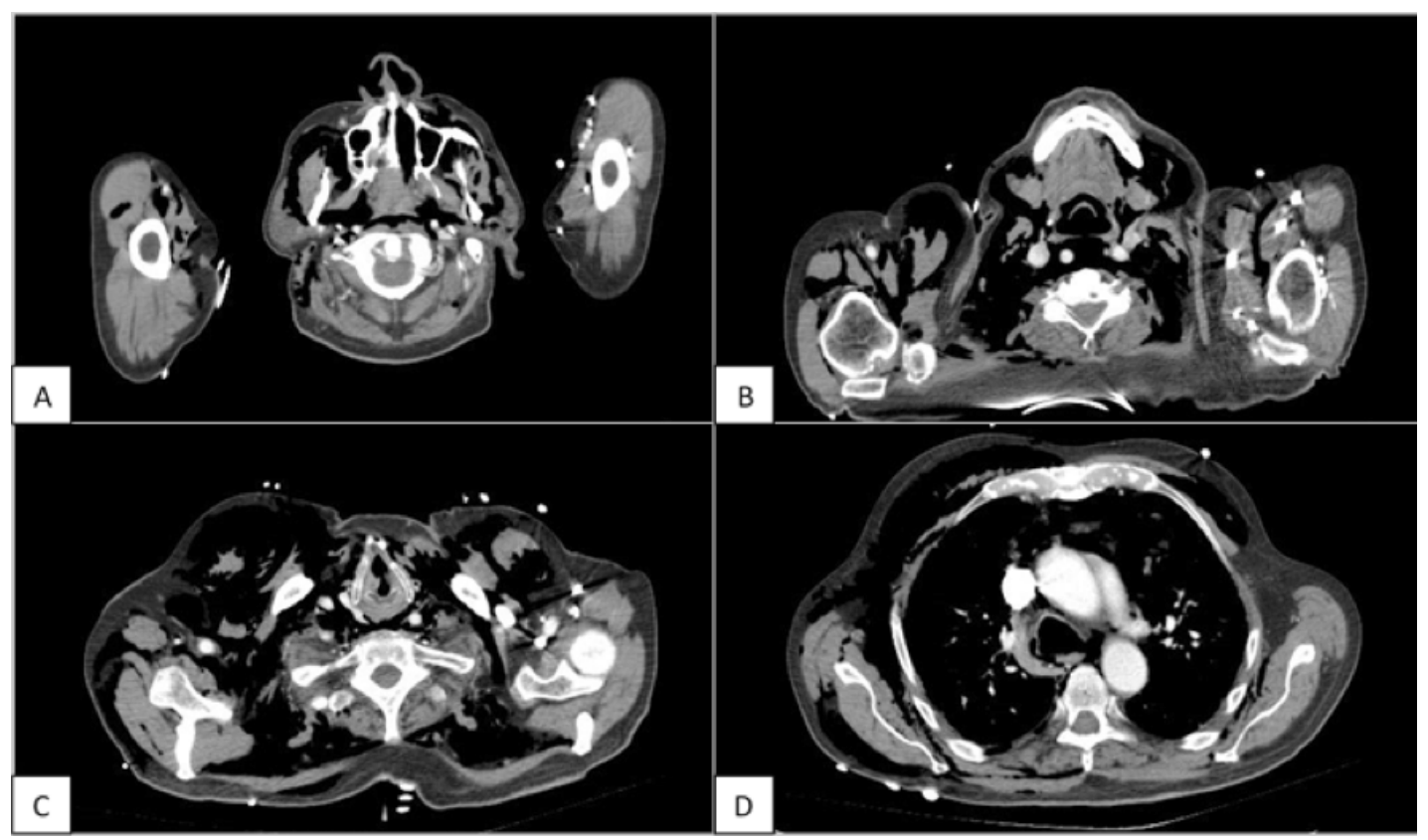

Figura 1. TC cervicotorácica que muestra extenso enfisema cervical en región maxilar (A), en zona retrofaríngea (B) y en región prelaríngea (C). Se puede apreciar presencia de aire en mediastino (D). 


\section{SÍNDROME DE HAMMAN: NEUMOMEDIASTINO ESPONTÁNEO QUE DEBUTA COMO ENFISEMA CERVICOTORÁCICO MASIVO. DESCRIPCIÓN DE UN CASO GARCÍA-CURDI F ET AL}

acertadamente cuando se presenta. Para el diagnóstico suele ser suficiente la radiografía de tórax, sin embargo, es necesario solicitar una TC si existen dudas diagnósticas. Se pueden realizar una broncoscopia y una esofagoscopia para descartar patología subyacente $[3,8]$.

Por lo general el tratamiento del NME, después de excluir cualquier causa grave, es conservador, combinando oxigenoterapia con tratamiento analgésico. En caso de sospecha de mediastinitis, se debe instaurar un tratamiento antibiótico intravenoso de manera urgente [2].

En casos en los que exista compresión de grandes vasos o de la tráquea, puede ser necesaria una videotoracoscopia o una toracotomía. Cuando exista un enfisema subcutáneo puede ser necesaria la incisión en la piel o incluso la inserción de un drenaje subcutáneo.

\section{CONCLUSIONES}

El neumomediastino debe ser tenido en cuenta en el diagnóstico diferencial de aquellos pacientes que sufren dolor torácico asociado a disnea. Por lo general, se requiere un tratamiento conservador con observación y seguimiento, aunque en ocasiones pueden surgir complicaciones graves que requieran una intervención urgente, incluso producir la muerte como en el caso de nuestro paciente.

Consideramos interesante la publicación de este caso clínico de NME, que debuta con dolor torácico, disnea y enfisema cervical subcutáneo sin un origen traumático, debido a lo llamativas que son las imágenes al diagnóstico.

\section{BIBLIOGRAFÍA}

1. Hamman L. Spontaneous mediastinal emphysema. Bull Johns Hopkins Hospital 1939; 64: 1-21.

2. Álvarez C, Jadue A, Rojas F, Cerda C, Ramírez M, Cornejo C. Neumomediastino espontáneo (síndrome de Hamman): Una enfermedad benigna mal diagnosticada. Rev Méd Chile 2009;137:104550.

3. Grapatsas K, Tsilogianni Z, Leivaditis V, Kotoulas $\mathrm{S}$, Kotoulas C, Koletsis E et al. Hamman's syndrome (spontaneous pneumomediastinum presenting as subcutaneous emphysema): A rare case of the emergency department and review of the literature. Respir Med Case Rep. 2017;23:63-5.

4. Macklin MT, Macklin CC. Malignant interstitial emphysema of the lungs and mediastinum as an important occult complication in many respiratory diseases and other conditions: an interpretation of the clinical literature in the light of laboratory experiment. Medicine. 1944;23:281-358.

5. Martín-Menjívar E, Vázquez Minero JC, Ferrero Balado E, Domíngez A. Efecto Macklin como causa de neumomediastino posterior a contusión torácica. Reporte de un caso. Trauma. 2007;10:5861.

6. Abad Royo JM, Batista Rocha L, Abad Domingo $\mathrm{Cl}$, González Eizaguirre M, Alonso Alonso L, Pérez Sánchez P. Enfisema cervical masivo espontáneo. ORL Aragón 2014;17(2):9-10.

7. Artal R, Agrega B, Serrano E, Sebastián JM, Alfonso JI, Valles H. Enfisema cervical subcutáneo: Una complicación rara tras maniobra de higiene bucodental. ORL Aragón 2008;11(1):18-20.

8. Gerazounis M, Athanassiadi K, Kalantzi N, Moustardas M. Spontaneous pneumomediastinum: a rare benign entity. J Thorac Cardiovasc Surg 2003;126:774-6. 